\title{
Concepción del alineamiento estratégico como principio de la gobernanza universitaria
}

\author{
ARMENIO PÉREZ MARTÍNEZ* \\ AIMARA RODRÍGUEZ FERNÁNDEZ** \\ Artículo de reflexión sobre la integración del alineamiento estratégico como principio de la gober- \\ nanza universitaria. \\ Recibido: 20 de abril de 2020 - Evaluado: 26 de junio de 2020 - Aceptado: 22 de julio de 2020 \\ Citar como: Pérez, A. y Rodríguez, A. (2021). Concepción del alineamiento estratégico \\ como principio de la gobernanza universitaria. Hallazgos, 18(35), 233-257. DoI: https://doi. \\ org/10.15332/2422409X.5689
}

* Doctor en Ciencias Económicas por la Universidad de Camagüey, Cuba. Coordinador del Departamento de Innovación y Calidad Universitaria, Universidad Laica Vicente Rocafuerte de Guayaquil, Ecuador.

Correo electrónico: aperezm@ulvr.edu.ec ORCID: https://orcid.org/0000-0002-9491-6938

** Doctora en el Programa de Aportaciones Educativas a las Ciencias Sociales y Humanas por la Universidad de Granada, España. Rectora de la Universidad Laica Vicente Rocafuerte de Guayaquil, Ecuador.

Correo electrónico: airodriguezf@ulvr.edu.ec

ORCID: https://orcid.org/0000-0002-6654-2588 


\section{Resumen}

El sistema de educación superior enfrenta nuevos retos que demandan significativos esfuerzos para garantizar su adecuado desempeño, lo que contribuye a la pertinencia social. En este contexto, la gobernanza universitaria desempeña un papel fundamental para obtener resultados superiores. Sin embargo, existen aspectos que afectan su funcionamiento desde una perspectiva sistémica. El alineamiento estratégico, como principio de gestión puesto en práctica en diferentes organizaciones, brinda una oportunidad de integración teórico-metodológica que contribuye al mejor funcionamiento del sistema de educación superior. El objetivo de este artículo de reflexión es argumentar la integración del alineamiento estratégico a la práctica de la gobernanza universitaria como un principio que garantiza la obtención de mejores resultados en beneficio de la sociedad. El paradigma metodológico utilizado es el cualitativo. La investigación es deductiva, desde una perspectiva analítica, sistémica y flexible. Los métodos utilizados son el análisis y la síntesis. Las conclusiones apuntan a la validez de la integración del alineamiento estratégico como principio de la gobernanza universitaria y la identificación de los factores de alineamiento que están presentes dentro del sistema de educación superior.

Palabras clave: Alineamiento estratégico; Educación superior; Gobernanza universitaria. 


\title{
Understanding of strategic alignment as a principle of university governance
}

\begin{abstract}
The higher education system faces new challenges that demand significant efforts to ensure its adequate performance, which contributes to social relevance. In this context, university governance plays a fundamental role in obtaining superior results. However, there are aspects that affect its functioning from a systemic perspective. Strategic alignment, as a management principle put into practice in different organizations, provides an opportunity for theoretical-methodological integration that contributes to the better functioning of the higher education system. The purpose of this reflection article is to argue for the integration of strategic alignment into the practice of university governance as a principle that guarantees better results for the benefit of society. The methodological paradigm used is the qualitative one. The research is deductive, from an analytical, systemic and flexible perspective. The methods used are analysis and synthesis. The conclusions point to the validity of the integration of strategic alignment as a principle of university governance and the identification of alignment factors that are present in the higher education system.
\end{abstract}

Keywords: Strategic alignment; Higher education; University governance.

\section{Concepção do alinhamento estratégico como princípio da governança universitária}

\section{Resumo}

O sistema de ensino superior enfrenta novos desafios que exigem significativos esforços para garantir seu adequado desempenho, o que contribui para a pertinência social. Nesse contexto, a governança universitária desempenha um papel fundamental para obter resultados superiores. Contudo, existem aspectos que afetam seu funcionamento sob uma perspectiva sistêmica. $\mathrm{O}$ alinhamento estratégico, como princípio de gestão colocado em prática em diferentes organizações, oferece uma oportunidade de integração teórico-metodológica que contribui para o melhor funcionamento do sistema de ensino superior. O objetivo deste artigo de reflexão é argumentar a integração do alinhamento estratégico à prática da governança universitária como um princípio que garante melhores resultados em prol da sociedade. O paradigma metodológico utilizado é o qualitativo. A pesquisa é dedutiva, sob uma perspectiva analítica, sistêmica e flexível. Os métodos utilizados são a análise e a síntese. As conclusões indicam a validade da integração do alinhamento estratégico como princípio da governança universitária e a identificação dos fatores de alinhamento que estão presentes no sistema de ensino superior.

Palavras-chave: Alinhamento estratégico; Ensino superior; Governança universitária. 


\section{Introducción}

La sociedad contemporánea se encuentra en un momento crucial de su existencia. Como nunca antes las condiciones que garantizan la continuidad de la especie humana han entrado en una situación tan crítica. Las instituciones sociales tienen que enfrentar esta situación con base en las potencialidades de los miembros que las componen. La Organización de las Naciones Unidas, a través de los Objetivos y Metas de Desarrollo Sostenible (ONU, 2015) ha estipulado las principales áreas de interés común para el desarrollo. Las universidades, como instituciones sociales, tienen que contribuir a la erradicación de la pobreza, el hambre, el analfabetismo, el trabajo infantil, la violencia contra la mujer, entre otras realidades. "La doble finalidad de la universidad desde su invención, a finales del siglo XI, es producir y difundir el saber que ella misma ha contribuido a crear" (Unesco, 2011, p. 97).

La política pública sobre educación superior en América Latina y en gran parte del mundo ha sido insuficiente para el desarrollo homogéneo de las universidades. Esta política ha sido más empleada por los gobiernos como elemento populista y electoral, que como compromiso real con las necesidades propias del sistema de educación superior y de la sociedad (De Melo, 2018; Alzate y Cardona, 2018). Los principales elementos de este tipo de política se encuentran vinculados al aumento del acceso y la elevación de la calidad, por consiguiente, se han descuidado otros aspectos esenciales como la financiación, la internacionalización, la investigación, la ubicación laboral, etc. Se coincide con Knobel y Bernasconi (2017) sobre el hecho de que estas políticas públicas son tardías, reactivas y carentes de sistematicidad. La educación superior mantiene tensiones con su entorno en lo referente al acceso, la pertinencia, la calidad y el financiamiento entre otros grandes temas.

La historia de las universidades latinoamericanas no puede ser contada sin hacer referencia a la Reforma de Córdoba. Este importante hecho histórico, con particular significación para las universidades, marcó la agenda de organismos y foros académicos en 2018. Reuniones de autoridades y académicos e importantes publicaciones acogieron debates y análisis sobre los retos de la educación superior. Sin embargo, pocos cambios se han materializado desde esa fecha hasta nuestros días. Lo que ha dejado el Centenario de la Reforma de Córdoba en materia de análisis sobre la realidad de las universidades es un renovado interés por el estudio del sistema universitario, buscando así elevar la pertinencia social según las carencias de cada región. Se aprecia un aumento de las investigaciones que abordan tanto el sistema como las instituciones de educación superior (IES) en artículos, con lo cual se 
incrementó el número de publicaciones sobre este tema incluidas en revistas científicas de reconocido prestigio.

Dentro de los temas se destaca la atención ofrecida a la gobernanza universitaria. Como nunca antes su importancia, actualidad y relevancia han sido puestas de manifiesto. En algunos casos como análisis teórico, en otros como estudios de las mejores prácticas; los avances teóricos y metodológicos en cuanto a la gobernanza universitaria encontraron un espacio y han generado eco en las investigaciones actuales que se desarrollan en las IEs, evidenciando así su relevancia y aplicabilidad (Sánchez y Castañón, 2019; Maldonado, Buenaño y Benavides, 2019; Garzón y Rodríguez, 2019; Krüger et ál., 2019).

Según Ashby (1963, p. 6), "aunque dedicados a la búsqueda del conocimiento, los universitarios han declinado hasta hace poco buscar el conocimiento sobre ellos mismos”. En el afán de perfeccionar el funcionamiento de las universidades, sus resultados, la gestión de sus recursos, entre otros elementos esenciales, la gobernanza universitaria emerge como uno de los temas investigativos más importantes en la última década (Scott, 2014; Brunner y Ganga, 2016; Ganga y Núñez, 2018; Pérez, Aguilar y Rodríguez, 2018).

La gobernanza universitaria ha tenido significativos avances como conocimiento científico, conformando un sistema monolítico aunque heterogéneo de saberes, metodologías y prácticas, desarrollado desde diversas perspectivas académicas interdisciplinarias, construido en beneficio de la interpretación de la complejidad inherente a los sistemas de educación superior. La integración del conocimiento ha dado como resultado el aumento del número de investigadores y publicaciones que se dedican a estos asuntos, así como de las autoridades universitarias que reconocen y aceptan las explicaciones que se brindan a los problemas internos desde esta perspectiva. Una idea útil al respecto enfatiza que "el buen gobierno de las universidades no es condición suficiente pero sí necesaria para asegurar una contribución efectiva de las universidades a su entorno" (Samoilovich, 2007, p. 69).

Diversos autores han reconocido la necesidad de continuar realizando investigaciones en materia de gobernanza universitaria (Ganga, 2014; Maldonado, Buenaño y Benavides, 2019; Alzate, Chaverra y Arango, 2020), desde la perspectiva de que "en el conocimiento sobre gobernanza universitaria es posible reconocer la desarticulación entre los resultados investigativos, su heterogénea naturaleza y su dificultad para la aplicación” (Pérez, Aguilar y Rodríguez, 2018, p. 58). Dentro de los temas que interesan, con particular significado al sistema universitario, se encuentran: la estructura institucional, los modelos de gestión aplicados, el acceso y la gestión del 
financiamiento, la selección de autoridades, el funcionamiento de los órganos decisores, entre otros. Estos temas armonizan con la investigación que se realiza desde la perspectiva de la gobernanza universitaria, concebida como un eje integrador interdisciplinario y multidimensional.

Sin embargo, no todo ha sido sencillo en estos temas de gobernanza. En la medida en que los retos sociales aumentan, el sistema de educación superior debe responder de manera ágil, precisa y decidida, marcando el rumbo del desarrollo integral de la sociedad. Varias investigaciones han demostrado la existencia de contradicciones entre los distintos niveles de organización del sistema de educación superior (Ganga, 2014; Ganga y Núñez, 2018), lo cual ha dado al traste con las intenciones de alcanzar resultados pertinentes y permanentes en el corto plazo. Por esta razón, es necesario realizar un ejercicio crítico y reflexivo dentro de las universidades, una valoración de la responsabilidad social universitaria, analizando las prácticas y los resultados, los impactos sociales, económicos, ambientales, académicos, etc.

Las ciencias económicas y la administración en particular han generado nuevas propuestas teóricas y metodológicas que contribuyen al mejor funcionamiento de las organizaciones de cualquier naturaleza (Stoner y Freeman, 1994). La dirección estratégica, la gestión de la calidad, la dirección por valores, el control de gestión, la gestión de capital humano, la logística, entre otras, son algunas de las propuestas desarrolladas en los últimos cincuenta años (Peters y Waterman, 1986; Ansoff, 1998; Ronda, 2007; Dolan, 2012; Scott, 2014).

El alineamiento estratégico es un principio del control de la gestión que ha puesto de manifiesto la herramienta gerencial conocida como "Cuadro de Mando Integral” (Kaplan y Norton, 2001; 2002; 2006). Los resultados de las aplicaciones de este principio en la práctica se encuentran evidenciados en múltiples organizaciones (Ballvé, 2000; Olve, Roy y Wetter, 2002; Kaplan y Norton, 2004; Naranjo, Mesa y Solera, 2005). En el contexto universitario, existe evidencia sobre los resultados positivos de la aplicación del alineamiento estratégico a la gestión institucional (Berrios, Castillo y Castro, 2009). Teniendo en cuenta estos referentes, se pretende fundamentar la integración del alineamiento estratégico a la aplicación de la gobernanza universitaria.

Por ello, el objetivo del presente artículo es argumentar dicha integración a la práctica de la gobernanza universitaria como un principio que garantiza la obtención de mejores resultados en beneficio de la sociedad. En esta línea de pensamiento, se ha realizado un estudio explicativo del vínculo teórico-metodológico entre la gobernanza universitaria y el alineamiento estratégico, a partir del análisis 
de experiencias en universidades latinoamericanas donde se han desarrollado investigaciones sobre estos temas. En el apartado teórico se ha realizado un análisis documental de los principales investigadores de estas temáticas en el contexto universitario. En consecuencia, se han aplicado métodos científicos como el análisis y la síntesis para la argumentación realizada. Esta contribución propone un avance en el conocimiento acerca de la gobernanza universitaria, generando una perspectiva interdisciplinaria para la búsqueda de alternativas que den solución a sus problemas fácticos. A la vez, adquiere relevancia y pertinencia por la utilidad que encierra en beneficio de las instituciones, las autoridades y la comunidad universitaria, dotando de cohesión interna y externa a las acciones directivas desde la gobernanza.

\section{Aspectos metodológicos de la investigación realizada}

El diseño metodológico propuesto resulta apropiado para el cumplimiento del objetivo de la investigación y el logro de su función social. Se realiza desde el paradigma cualitativo, siendo consecuentes con la búsqueda y el análisis de la información, así como con la esencia ética y la veracidad científica. Se emplea una perspectiva analítica, interpretativa y crítica sobre un tema específico (Sánchez, 2011), garantizando las proposiciones con respaldo teórico, en fidelidad hacia la lógica del fenómeno estudiada (Laguna et ál., 2007). En este caso, se analizó la relación entre la gobernanza universitaria, entendida como enfoque de gestión del sistema de educación superior, y el alineamiento estratégico, comprendido como un principio de gestión probado en varias empresas y organizaciones sociales.

Para la selección de la información, se realizó una búsqueda bibliográfica a partir de la identificación de autores destacados en estos temas, entre los cuales se incluyeron los de mayor reconocimiento en el contexto académico. Asimismo, se efectuó el análisis sistemático de la información, lo que permitió la identificación de las posturas comunes entre los diversos autores. Con posterioridad, se empleó la síntesis para elaborar, desde un punto de vista interpretativo, una novedosa perspectiva de las categorías analizadas, lo cual permitió integrar teórica y metodológicamente el alineamiento estratégico como principio de la gobernanza universitaria. 


\section{Una mirada a la educación superior contemporánea}

A medida que los problemas sociales y económicos que enfrenta la humanidad se agudizan, la importancia de las instituciones de educación superior ha tomado un papel más relevante para las distintas organizaciones internacionales. La Unesco planteó en la Declaración Mundial sobre la Educación Superior para el siglo xxi, en su artículo 6, letra (b):

La educación superior debe reforzar su servicio a la sociedad y en especial sus actividades para eliminar la pobreza, la intolerancia, la violencia, el analfabetismo, el hambre, la degradación ambiental y la enfermedad utilizando principalmente enfoques transdisciplinarios e interdisciplinarios en el análisis de los temas y los problemas. (Unesco, 1998, p. 7)

La importancia actual de las universidades para el desarrollo sostenible de la sociedad ha sido señalada por importantes autores y organizaciones internacionales (Mendoza, 2011; Unesco, 2011). La sociedad del conocimiento ubica a la universidad como una institución social de vanguardia, determinante en la solución de los más disímiles problemas sociales que actualmente aquejan a la humanidad, además de su papel protagónico en la transmisión social del conocimiento en función del desarrollo social. Si bien existen otro tipo de instituciones científicas y tecnológicas que tienen la misión de generar conocimiento para su aplicación tecnológica, las universidades también contribuyen a este objetivo con capital humano, condiciones tecnológicas y recursos financieros (Calderón, 2009; García, 2018; Hueso, 2019).

Las universidades son un pilar del desarrollo económico y social de las regiones. Desde esta perspectiva, cobra sentido que las mejores se encuentren en los países más desarrollados (Brunner y Ganga, 2016; Pérez, Aguilar y Rodríguez, 2018). No existen dudas sobre el hecho de que las universidades concentran un gran potencial para cualquier país, potencial intelectual y tecnológico capaz de transformarse en fuerza productiva, acción política y crecimiento económico. Ellas garantizan una perspectiva del desarrollo comprometida con un enfoque de ciencia y tecnología, en función de la sociedad y asociada al papel de las ciencias sociales y humanísticas (Murcia y Gamboa, 2014).

Históricamente, las universidades han sido consideradas como instituciones autónomas, académica y administrativamente, por ello reciben ciertos beneficios en cuanto a la gestión de sus recursos. Las universidades son, en ese sentido, entidades diversas y complejas. Ginés (2018) plantea que existe gran diversidad de profesores, 
estudiantes, autoridades, métodos de enseñanza, diseño de procesos, indicadores de gestión y estándares, propios de la evolución de este sistema, bajo el amparo de los principios de autonomía y de responsabilidad social, lo que evidencia la complejidad y diversidad del sistema de educación superior. El modo en que se vinculan las universidades con las distintas instituciones del entorno es único, dependiendo del país y de los organismos de control, así como de las características de las empresas, familias, comunidades y otras organizaciones. Se puede apreciar que una importante variedad de modelos de gestión, estrategias y enfoques administrativos son aplicados en la dirección de las IEs, pluralidad que se justifica por la autonomía de las instituciones y las regulaciones existentes en cada país. Autores como Berrios, Castillo

y Castro (2009) y Almuiñas y Galarza (2015) han enfatizado esta idea. Sin embargo, es necesario reconocer que la aplicación de la dirección estratégica, la dirección por valores, el control de gestión, entre otras, son extraídas de la práctica empresarial, lo cual requiere un análisis y adecuación para su aplicación en el sistema universitario (Ganga, Pérez y Mancilla, 2018).

En el funcionamiento y dirección de las IEs confluyen varios constructos y metodologías propios de las ciencias sociales afines como son la administración, la economía, la psicología, la sociología, entre otras. Según Ganga (2014):

\footnotetext{
Las universidades son lugares donde las ideas y los valores están profundamente integradas con las estructuras, funciones, roles y culturas, y que se trata de un sistema de actos laborales, económicos y tecnológicos concatenados en clausura operacional, que se constituye como unidad en el espacio de relaciones humanas. (p. 15)
}

Todas estas transformaciones recientes dentro del sistema universitario han puesto en evidencia la necesidad de realizar investigaciones sobre el gobierno de las universidades, en la medida en que se reconoce que las distintas formas de gobierno universitario no pueden ser imitadas textualmente de otras experiencias prácticas, sino que requieren de una proyección y construcción propias, desde las necesidades de las instituciones y las metas que se hayan establecido para su desarrollo.

\section{Antecedentes sobre la gobernanza universitaria}

En la esencia del concepto de gobernanza universitaria se encuentra la idea de que esta: 
[...] apunta a la manera en que las universidades se encuentran organizadas y estructuradas, y al modo como ellas son administradas desde la óptica de su gobierno y gestión; incorporando además el diseño que tienen para establecer manejar sus vínculos y relaciones con entes y entidades del entorno. (Ganga, 2014, pp. 15-16)

Este concepto distingue entre componentes como gobierno y gestión de procesos, organización y estructura. Por esta razón, brinda la posibilidad de desarrollar una postura compleja e interdisciplinaria, mediante la creación de espacios para la investigación de estas características y la generación de vínculos entre estos.

Son, entonces, varios los elementos que se orientan hacia la transformación de las universidades, la manera en que estas se relacionan con el entorno y se modifican, y por ende, la forma como va evolucionando el gobierno universitario. Las principales modificaciones en las demandas se encuentran vinculadas a los procesos de aseguramiento de la calidad, la gestión financiera y los procesos de ingreso y egreso de las universidades, así como a la presión de grupos de interés internos y externos (Viancos y Ganga, 2020). En ese sentido, la universidad es una institución que se debate entre los grupos internos (docentes, estudiantes, autoridades y funcionarios), que tradicionalmente han generado fuertes enfrentamientos desde la Reforma de Córdoba de 1918, y los grupos externos, que reconocen los resultados de las instituciones con relación a sus intereses particulares, desde los cuales pueden ofrecer beneficios o sanciones.

Uno de los temas que más ha afectado el avance del conocimiento sobre gobernanza universitaria es su reducción a la investigación sobre gestión universitaria, es decir, a lo relacionado con el funcionamiento interno de las IEs. Este enfoque reduccionista ha fracasado, ya que limita la verdadera función de la gobernanza universitaria para potenciar la integración del sistema de educación superior y responder de manera más efectiva a las demandas sociales. Ampliar esta perspectiva de análisis a las estructuras que se encuentran dentro de cada nivel, las relaciones que se establecen en la práctica y las funciones de cada estructura que realmente abarcan la gobernanza universitaria, permite que dicho análisis se aborde desde una postura interdisciplinaria, con equipos de profesionales que contribuyan al estudio de este importante tema.

Se considera que el conocimiento generado sobre gobernanza universitaria ha crecido con solidez académica en la última década gracias a tres elementos esenciales. En primer lugar, la agudización de los problemas sociales, que demandan soluciones 
urgentes desde lo académico (profesores, alumnos y egresados) con la aplicación de las potencialidades que brindan las nuevas tecnologías. Estas demandas deben ser atendidas desde las funciones sociales que cumple la universidad contemporánea: docencia, investigación y vinculación con la sociedad.

En segundo lugar, la importancia para la sociedad del óptimo funcionamiento del sistema de educación superior, manteniendo un interés político y social sobre las contradicciones internas del sistema y la respuesta a las demandas de accesibilidad, aseguramiento de la calidad, internalización, financiamiento, etc. En tercer lugar, el propio funcionamiento de las IES a la hora de desarrollar su estrategia, cumplir con su encargo social y conducir a la organización al logro de sus metas, aun contando con limitados recursos y fuerzas antagónicas.

Los resultados científicos en torno a la gobernanza universitaria tienen que contribuir a la agenda de los principales tomadores de decisión en materia de política pública sobre la educación superior, los cuales deben sortear al mismo tiempo las exigencias sociales relacionadas con el acceso, la calidad y la pertinencia para garantizar el aumento de conocimientos en función del desarrollo social. Al hablar del conocimiento acumulado en torno a la gobernanza universitaria, se hace referencia a importantes aportes realizados desde distintos ámbitos del saber, desde una perspectiva dialéctica e integradora.

Dentro de los principales elementos que afectan la gobernanza universitaria en la actualidad se encuentran el efecto pinball (asociado con elementos burocráticos, que entorpecen el óptimo funcionamiento de las IEs y la obtención de mejores resultados); el conflicto del doble papel (relacionado con la teoría de la agencia) y el conservadurismo y tradicionalismo. También han sido identificado otros factores (Ganga, Pérez y Mancilla, 2018, p. 3), tales como:

- Ausencia de valores y de cognición directiva.

- Operatividad excesiva en las decisiones (decisiones tácticas por encima de las estratégicas).

- Falta de perspectiva estratégica.

- Desconocimiento del territorio en el que se sitúa la universidad.

- Carencia de alineamiento entre la proyección estratégica y el trabajo operativo.

- Separación de funciones y compartimentación estancada en la estructura organizacional.

- Ausencia de autogestión para obtener financiamiento. 
- Desarrollo desigual interno de las Facultades y los Departamentos (falta de mirada sistémica).

- Ineficiencia de los órganos colectivos de la dirección universitaria.

Al hablar de gobernanza universitaria, es necesario reconocer la existencia de tres niveles de organización social, además de una red indisoluble de relaciones entre estos (Pérez, Aguilar y Rodríguez, 2018): el Gobierno o el sistema de educación superior, dedicado a organizar la política pública y los fines del sistema en relación con la sociedad; los organismos de control, que materializan las políticas públicas trazadas por el Gobierno y, al mismo tiempo, se encargan de concretar la regulación de las instituciones; y por último, las instituciones mismas, que es donde se relacionan los actores (estudiantes, docentes, autoridades y administrativos), los procesos y los resultados internos. Estos tres elementos interactúan de manera particular en cada país, región y universidad, por ello la gobernanza universitaria está generando constantemente nuevas formas de relación y demanda de investigaciones más novedosas y pertinentes, con explicaciones y predicciones sobre esta relación de distintos elementos y niveles.

Desde la perspectiva teórica, se identifican además dos retos sobre el conocimiento de la gobernanza universitaria. En primer lugar, se encuentra la necesidad de integrar con otras categorías científicas el análisis del objeto de estudio comprendido como el sistema de educación superior, lo cual significa generar un espacio de diálogo con enfoque interdisciplinar, que ofrezca sólidos referentes teóricos, capacidades de integración en redes de académicas, y la posibilidad de creación de nuevos núcleos de investigación con perspectiva integradora. Este es un reto vigente para toda la ciencia actual comprometida con el análisis de la realidad de manera integrada, sin divisiones ni antagonismos, en búsqueda de que se reconozca el carácter complejo de los análisis que se realizan.

Un segundo reto teórico es el avance de un cuerpo conceptual propio con capacidad de integrar el conocimiento que ha sido generado desde otras ciencias sociales afines como la administración, la psicología o la sociología, y que a la vez sea motivante para que continúe su análisis a partir de proyectos de investigación derivados del conocimiento de la gobernanza universitaria.

Esto requiere una doble valoración: por un lado, la importancia práctica del conocimiento generado, lo cual se ha alcanzado a partir de la aceptación que tienen los resultados de gobernanza universitaria entre las autoridades del sistema de educación superior (Ganga, 2014; Ganga y Núñez, 2018) y de una mayor visibilización de 
estos resultados; y por otro lado, la calificación dentro del espectro de las ciencias sociales, aceptando que existen conceptos y metodologías propias (Ganga, Pérez y Mancilla, 2018; Naranjo, Mesa y Solera, 2005), así como investigadores y espacios para la difusión de este conocimiento. Se requiere por lo tanto integrar en núcleos teóricos que se relacionen entre sí, dando explicaciones al funcionamiento de la compleja realidad que se estudia y logrando un mejor funcionamiento según las demandas sociales.

Estos dos desafíos en el plano teórico garantizan la evolución futura de este conocimiento, la generación de nuevos saberes que irán complementando los actuales y la formulación de nuevas contradicciones a partir de la demanda social. Un elemento significativo es el carácter pertinente y comprometido de la gobernanza universitaria, siempre en busca de brindar soluciones a problemas sentidos de la comunidad universitaria y de todo el sistema de gobierno, la política pública y los organismos de control. Si en otras ciencias se puede distinguir con facilidad el desarrollo de investigaciones futuristas o que generan un conocimiento para posibles escenarios, la gobernanza universitaria, por su parte, se encuentra vinculada al estudio del presente y la cotidianidad de las universidades y su entorno.

Los retos no se quedan solamente en el plano teórico, ya que la mayor parte del conocimiento de la gobernanza universitaria emerge de investigaciones prácticas. Por lo tanto, los investigadores no deben perder de vista estas contradicciones metodológico-prácticas que se generan a la hora de estudiar el gobierno del sistema de educación superior, tales como el conflicto del doble papel y el efecto pinball. Dentro del análisis realizado se aprecian tres aspectos metodológico-prácticos que son desafíos para este conocimiento. En primera instancia, se valoran altamente los resultados actuales de autores que evidencian determinadas áreas requeridas de investigación minuciosa para desentrañar su particular funcionamiento. Dentro de estas nuevas unidades de análisis propuestas se encuentran:

- Pertinencia de la política pública en materia de educación superior, según las peculiaridades del desarrollo de cada país y región.

- Funcionamiento de los organismos de control de la política pública; creación y validación de estándares e indicadores; puesta en práctica de sistemas de aseguramiento de la educación superior.

- Tipologías de gobiernos para las IEs; búsqueda de financiamiento; pertinencia de la formación e investigación universitaria desde la perspectiva de la gobernanza universitaria. 
- Estructuras, liderazgo, coordinación, colaboración, representatividad en los órganos decisores de las IEs.

- $\quad$ Efecto pinball, conflicto del doble papel, conservadurismo y tradicionalismo.

- Un segundo desafío metodológico-práctico se asocia al funcionamiento de cada nivel, pues existen contradicciones dentro de cada subsistema del sistema de educación superior, lo que origina mal funcionamiento y la imposibilidad de cumplir con la misión social que tienen las IEs. Dentro de cada subsistema se pueden enunciar:

\section{Gobierno}

El Gobierno es el encargado de trazar la política pública en materia de educación superior, a partir de las demandas sociales y de las necesidades de desarrollo de cada país. Dentro de las contradicciones presentes en el Gobierno se encuentran:

- Necesidad de mecanismos que generen mayor rapidez en las decisiones relacionadas con las modificaciones a la política pública y su marco legal.

- Carencias en el marco jurídico que impiden la pertinencia y el desarrollo sostenible del sistema de educación superior, así como de sus aportes al entorno y al reconocimiento por parte de la sociedad.

- Incapacidad de garantizar los fondos necesarios para el desarrollo sostenible de la educación superior. Esto no significa que el financiamiento deba ser totalmente público, sino que actualmente no se garantiza el financiamiento necesario, sea cual fuera su fuente de procedencia.

- Pérdida del carácter estratégico en su gestión de situaciones puntuales, sin garantizar el enfoque prospectivo y rector de la política pública.

- Insuficiente visibilización de las demandas de los grupos minoritarios y tradicionalmente excluidos en materia de educación superior, en la política pública.

\section{Organismos de control}

Los organismos de control son los encargados de gestionar y controlar la política pública en materia de educación superior. Su papel regulador exige el constante vínculo con las IEs, la sociedad y el Estado. Algunas contradicciones presentes en este subsistema son: 
- Ineficiencia de los mecanismos de control de la política pública; desactualización de indicadores; carencia de personal calificado.

- Lentitud en la interpretación de las demandas sociales y de la política pública.

- Excesiva aplicación burocrática de los mecanismos de evaluación de la política pública.

- Desarticulación con las IEs.

\section{Instituciones de educación superior}

Las IES centran la atención principalmente de la sociedad, en la medida en que son las encargadas de ejecutar la política pública en materia de educación superior. A la vez, cumplen con las funciones sociales de docencia, investigación y vinculación con el entorno. Algunas de sus limitaciones se manifiestan en:

- Funcionamiento de los órganos de dirección colectivos; representatividad de los actores universitarios.

- Papel de los directivos; elección y evaluación de desempeño.

- Los resultados institucionales y su relación con el entorno.

- Coherencia interna en la planeación estratégica de las iEs y los planes operativos dentro de las unidades estructurales (departamentos, cátedras, áreas, etc.).

El tercer desafío metodológico-práctico aparece como resultado de la integración de los distintos niveles dentro del sistema, reconociendo la importancia de cada uno de ellos, su carácter autónomo y de responsabilidad ante la sociedad. Lo que cada subsistema pueda aportar al óptimo funcionamiento del sistema de educación superior debe ser adecuadamente integrado y contextualizado dentro de los demás subsistemas. Por ejemplo, las actualizaciones en la política pública tienen que promover rápidas transformaciones en el equipamiento evaluativo de los organismos de control (metodologías, indicadores, personal evaluador, etc.), de modo tal que se posibilite captar el análisis proveniente desde las IEs, a partir de su autonomía responsable. Las brechas dentro del sistema corresponden a insuficiencias dentro de la red de relaciones establecidas entre los distintos niveles. Se aprecia que las tensiones presentes dentro del sistema (internas y externas) va a generar una respuesta integrada dentro de los distintos subsistemas para no generar caos y desorden, lo cual contribuye a la creación de una imagen errónea del funcionamiento del sistema, al no encontrarse en coherencia las demandas establecidas en los distintos niveles del sistema de educación superior. 
Los desafíos metodológico-prácticos están latentes en la actualidad. Es incuestionable que las IEs tienen que realizar grandes esfuerzos para lograr estabilidad en todo nivel, además de que deben cumplir con las solicitudes y exigencias de la política pública y de los organismos de control. Sin embargo, una de las carencias funcionales del sistema es el insuficiente alineamiento interno, debido a las brechas entre lo que se espera y lo que ocurre en realidad, entre la política pública trazada y el desempeño de las instituciones, porque hace falta ajustar los componentes y mecanismos de regulación e integración respecto a las necesidades actuales. Todo esto en conjunto genera insatisfacción de los grupos de interés y falta de efectividad en el uso de los recursos.

Las respuestas pueden surgir aquí desde varias propuestas interdisciplinarias. La posición que se sustenta en este artículo es la búsqueda de categorías científicas, con elevado carácter práctico, que generen un contexto capaz de captar estas variaciones dentro del sistema y ajustar los cambios de la manera más rápida y homogénea posible en todos los niveles. Para ello, una respuesta posible a esta aspiración es la aplicación del alineamiento estratégico.

\section{Criterios esenciales sobre el alineamiento estratégico}

En este ámbito de análisis surge la importancia de la investigación sobre el alineamiento estratégico, como forma para obtener resultados más pertinentes para el sistema de educación superior (Naranjo, Mesa y Solera, 2005; Alzate, Chaverra y Arango, 2020). Al considerar la perspectiva del alineamiento estratégico dentro de la gobernanza universitaria, es más viable que los objetivos trazados se puedan concretar en resultados en todos los niveles; esto significa que si se logran alinear los objetivos y las acciones de los tres niveles de organización del sistema de educación superior (Gobierno, organismos de control e instituciones), se obtendrán respuestas de manera integral, respetando así la autonomía de las universidades.

Los estudios realizados en ciencias administrativas sobre el funcionamiento de las organizaciones ofrecen aportes respecto a la gestión eficiente de los recursos para obtener resultados superiores (Peters y Waterman, 1986; Stoner y Freeman, 1994; Ansoff, 1998). Sin embargo, en la implementación de la dirección estratégica se ha demostrado que los principales problemas se encuentran en la ausencia de compromiso y en la falta de alineamiento de los objetivos y las metas con las tareas cotidianas de cada elemento dentro de la organización (Ronda, 2007, p. 19). 
Para Kaplan y Norton (2006), "se identifica el alineamiento como un componente explícito del proceso de gestión. La ejecución de la estrategia requiere del más alto nivel de integración y de trabajo en equipo entre las unidades y los procesos organizativos” (p. 301). Así, el alineamiento estratégico está asociado a técnicas de dirección de sistemas y organizaciones que vinculan a todas las partes integrantes. En este sentido, es un factor que garantiza la participación.

La relevancia del alineamiento estratégico para el óptimo desempeño de los sistemas y de las organizaciones es reconocida gracias a los prestigiosos resultados de investigaciones internacionales (Ballvé, 2000; Olve, Roy y Wetter, 2002; Kaplan y Norton, 2000; 2001; 2002; 2004; 2006). La congruencia entre las aspiraciones y los deseos de la sociedad, su formulación en la política pública, su traducción a indicadores de gestión e indicadores de resultados y su puesta en práctica por las instituciones, garantizan la obtención de resultados esperados desde la participación y el compromiso de todo el sistema de educación superior (Kaplan y Norton, 2000).

Según Dolan (2012), "lograr el alineamiento es un proceso de dos fases: la primera es la identificación y corrección de la falta de alineamiento. La segunda es crear el alineamiento con mecanismos de refuerzo reales" (p. 123). Este criterio refuerza la consideración de que es necesario identificar los elementos que obstaculizan el alineamiento estratégico, que pueden ser personales, estructurales y legales según sea su naturaleza. A partir de esta definición se buscan alternativas para minimizar su impacto en la aplicación del alineamiento estratégico.

Los autores señalados anteriormente definen varios factores de alineamiento estratégico que contribuyen al éxito empresarial (Kaplan y Norton, 2006, p. 95).

- Alineamiento entre la sede central y las sedes operativas.

- Alineamiento de las unidades internas de soporte y servicios.

- Alineamiento de las organizaciones externas.

- Alineamiento de la estructura con la estrategia.

- Alineamiento entre las perspectivas del cuadro de mando integral.

- Alineamiento del factor humano con la estrategia.

- Alineamiento de los sistemas de planificación y control. 


\section{Gobernanza universitaria y alineamiento estratégico: una alianza para garantizar resultados}

Los factores de alineamiento se consideran válidos para el análisis y el desarrollo de investigaciones comprometidas con las transformaciones sostenibles que requiere la sociedad contemporánea. Sin embargo, son insuficientes para generar el funcionamiento armónico del sistema de educación superior, debido a que dentro del mismo sistema se reconocen otros componentes de coherencia interna, no considerados en esta propuesta, que desde el punto de vista metodológico resultan igualmente determinantes.

Uno de los elementos que llama la atención, por su valor metodológico y por su aporte a la solución de las contradicciones en la práctica, es "la fundamentación de los factores estratégicos que necesariamente tienen que contar con un alineamiento, así como la definición y uso de los mecanismos de control de gestión, integrados al procedimiento propuesto, para comprobar la efectividad de los resultados propuestos" (Pérez, 2013, p. 69). Tal planteamiento evidencia la necesidad de definir y enunciar con claridad tanto los resultados como sus mecanismos de evaluación y el procedimiento diseñado para alcanzarlos, lo cual implica un enfoque más orientado al proceso que al resultado, más participativo, a la vez que se van inspeccionando las distintas etapas del proceso de gestión. Estos elementos resultan sumamente significativos en la fundamentación del alineamiento estratégico integrado en las prácticas de gobernanza universitaria.

El alineamiento estratégico como principio para la gestión del sistema de educación superior se establece para fomentar el análisis constante de todos los elementos integrantes del sistema, por consiguiente, se tendrían que visualizar desde cualquier nivel los aportes hechos para la obtención de los resultados esperados. Significa que la comunicación, coordinación y colaboración tienen que estar presentes, compartiendo información de manera precisa y oportuna, aportando a los debates y generando compromiso.

El alineamiento estratégico tiene que asumirse como una condición base de la gobernanza universitaria, ya que permite fortalecer:

[...] la relación estrategia-cultura organizacional, permitiendo la potenciación de los resultados organizacionales, a partir del compromiso de los sujetos en todos los niveles del sistema de educación superior; por lo tanto, puede convertirse en un factor de alineamiento y una guía para materializar las transformaciones que se realizan en el sistema. (Dolan, 2012, p. 42) 
Este concepto cuenta con suficiente reconocimiento académico y aval de aplicación práctica para convertirse en un principio clave dentro del sistema de educación superior y, a la vez, formar parte del objeto de estudio de la gobernanza universitaria, garantizando así una solución fáctica a algunos de los problemas teóricos, metodológicos y prácticos que se encarga de estudiar, ya que permite la integración del sistema y la obtención de resultados para los grupos de interés social y los actores de la comunidad universitaria.

Los elementos fundamentales por considerar en la propuesta de alternativas metodológicas para el mejoramiento del desempeño del sistema de educación superior como un todo son: el reconocimiento del alineamiento entre la política pública y el desempeño de las IEs, el empleo de los mecanismos reguladores asociado a las prácticas institucionales, y los objetivos de desarrollo autónomo y responsable, básicos para la obtención de resultados sociales adecuados.

Es necesario tener claridad en la definición de las metas esperadas, las categorías rectoras de la estrategia como sistema, las expectativas para cada uno de los niveles, las estrategias armónicamente estructuradas y los enunciados de los desempeños esperados en cada uno de los niveles, de esto depende poder generar una arquitectura coherente entre los elementos que integran el sistema de educación superior.

Guiados por el análisis práctico de experiencias en la aplicación del alineamiento estratégico en instituciones de educación superior (Naranjo, Mesa y Solera, 2005; Pérez, Naranjo y Mesa, 2017; Yera, Septién y Naranjo, 2019) y por el estudio teórico pormenorizado de esta metodología, se establecen los factores de alineamiento estratégico que se consideran indispensables para lograr su aplicación en la gestión del sistema de educación superior. Resulta importante en este sentido aclarar que en los sistemas y las organizaciones actúan factores de alineamiento necesarios para lograr los resultados a los que se aspira (Kaplan y Norton, 2006). Por ello, es indispensable reconocer que el sistema de educación superior se encuentra inmerso en un suprasistema de relaciones sociales, con ciertas particularidades y aportes para el óptimo funcionamiento de la sociedad. Siguiendo esta línea de análisis (Pérez, 2013), se aprecian tres momentos fundamentales de alineamiento estratégico:

- Alineamiento externo. El Estado, el sistema económico y empresarial, la sociedad civil, el sistema nacional o territorial de desarrollo tecnológico y científico, fundamentalmente, son los elementos del contexto que se relacionan con el sistema de educación superior y que determinan su éxito. Cada uno de estos elementos desempeña un papel particular y se integra en una red de relaciones en 
la cual el sistema de educación superior necesita captar recursos para desarrollar conocimiento y soluciones pertinentes.

- Alineamiento interno. La estructura, el presupuesto, los sistemas, los procesos, la logística propia del sistema, se convierten en insumos de su subsistencia, entre otros elementos que pueden influir en el logro de los objetivos, a partir del empleo adecuado de recursos internos. En esencia, la gobernanza en las IEs tiene que garantizar la coherencia en la gestión de las distintas áreas, para que las relaciones entre estas sean mutuamente beneficiosas y aporten al logro de los objetivos y las metas propuestas en la planeación estratégica universitaria.

- Alineamiento metodológico. Considera aquellos factores de alineamiento que intervienen directamente en la coherencia interna del proceso estratégico del sistema y de cada uno de sus niveles en particular.

A efectos de la presente propuesta, se plantean los siguientes enunciados de alineamiento estratégico que pueden resultar de mayor interés, dado que garantizan la coherencia entre la gestión del sistema y los resultados esperados, a partir de la implicación de los integrantes:

1. Alineamiento entre la política pública y las demandas sociales, así como de los grupos internos de las IEs. Garantiza que la política pública aprobada conduzca hacia la solución, o al menos visualice la necesidad de resultados que tiendan a minimizar o enfrentar demandas sociales relacionadas con el desarrollo económico, político, tecnológico, académico y social.

2. Alineamiento entre la política pública y el funcionamiento de los organismos de control. Genera confianza hacia la sociedad en su conjunto; al mismo tiempo, se traduce en un elemento de justicia para las IEs. Garantiza respeto por la autonomía responsable, si bien se necesitan pautas de evaluación claras por parte de las IES, para obtener resultados pertinentes y necesarios en el desarrollo conjunto de la sociedad.

3. Alineamiento entre la política pública y el funcionamiento de las IEs. Para que los tomadores de decisión de la política pública aseguren el desarrollo de una educación superior pertinente, autónoma y responsable.

4. Alineamiento entre las categorías del diseño estratégico en cada nivel del sistema de educación superior. En la medida en que todo diseño estratégico, desde el punto de vista metodológico, realiza un análisis de las condiciones externas e internas, identifica el marco legal regulatorio y propone estrategias 
e indicadores de gestión y resultados. Por lo tanto, el diseño estratégico debe respetar la coherencia entre estos elementos, consolidando una estrategia que genere resultados sociales y promueva la autonomía universitaria.

5. Alineamiento entre los objetivos de las IEs y el desempeño de sus entes decisores. Los órganos decisores colectivos deben perfilar su funcionamiento según los objetivos trazados por las IES; de la misma manera, las autoridades tienen que coadyuvar a la consecución de los objetivos para promover una relación sistémica entre la estrategia y los elementos de la cultura organizacional (creencias, normas, actitudes y valores).

Estos alineamientos mencionados no agotan la realidad. En la práctica pueden aparecer y ser objeto de análisis otros factores, lo cual enriquece la comprensión sobre el funcionamiento sistémico de la comunidad universitaria, así como su papel determinante en el desarrollo social.

\section{Conclusiones}

El sistema de educación superior a nivel internacional tiene un carácter determinante para la supervivencia y desarrollo en el futuro de la humanidad, por eso es constantemente convocado para la búsqueda de la solución a los principales problemas sociales. Luego las instituciones de educación superior demandan procesos de gestión que garanticen el cumplimiento de su encargo social.

La gobernanza universitaria contribuye a la disminución de las brechas que puedan generarse entre el gobierno, los organismos de control y las universidades para obtener los resultados demandados por la sociedad. Por ello, el desarrollo de este campo de investigación necesita avanzar teórica y metodológicamente para abordar los problemas prácticos que enfrenta, apoyándose en los aportes de otras ciencias sociales y a la vez colaborando con ellas, lo que significa una perspectiva interdisciplinaria y compleja en las investigaciones.

La coherencia entre los niveles organizacionales del sistema de educación superior requiere de continuos acercamientos, de diálogos, incluso de la revisión de las decisiones, para poder garantizar la inclusión de las demandas sociales en la política pública. Asimismo, precisa que se planteen indicadores que realmente evalúen el cumplimiento de dicha política y las exigencias sociales de desarrollo, así como 
la consolidación de las estrategias y prácticas de las IES que materializan la política pública, pues esto posibilita el abordaje de procesos de acreditación de sus funciones formativas, investigativas y de vinculación con la sociedad.

En esta sintonía, el alineamiento estratégico emerge como un principio de gestión capaz de integrar estos intereses desde puntos de vista teóricos y metodológicos. En la práctica, es un principio de la gestión del sistema de educación superior, un pilar en el logro de sus objetivos y una garantía para mantener el ajuste estructural y funcional del sistema como un todo, partiendo de la comprensión del papel de los distintos niveles organizativos dentro del sistema.

Los factores de alineamiento estratégico identificados constituyen una guía para la aplicación de este principio dentro de la gobernanza universitaria. Se considera sin embargo que pueden establecerse otros factores adicionales, que fomentarán el interés por su aplicación y contribuirán al aumento del conocimiento científico en este tema.

\title{
Sobre los autores
}

\begin{abstract}
Armenio Pérez Martínez. Doctor en Ciencias Económicas por la Universidad de Camagüey, Cuba. Coordinador del Departamento de Innovación y Calidad Universitaria de la Universidad Laica Vicente Rocafuerte de Guayaquil, Ecuador. Investigador en gobernanza universitaria, gestión de la calidad, gestión universitaria, teoría económica, psicología social. Publicaciones recientes: "La responsabilidad social universitaria en la dirección estratégica de las universidades" (2017), "Nueva gobernanza universitaria: enfoque omniabarcante para estudiar el gobierno en universidades latinoamericanas" (2018), "Paradigmas emergentes en la gobernanza universitaria: una aproximación teórica" (2018), “Gobernanza universitaria y valores: la función control en la gestión universitaria” (2018), “Tipología de los sistemas de aseguramiento de la calidad basado en la autonomía y la Responsabilidad Social Universitaria” (2020).
\end{abstract}

\begin{abstract}
Aimara Rodríguez Fernández. Doctora en el Programa de Aportaciones Educativas a las Ciencias Sociales y Humanas por la Universidad de Granada, España. Rectora de la Universidad Laica Vicente Rocafuerte de Guayaquil, Ecuador. Investigadora en temas sobre gobernanza universitaria, gestión de la calidad, gestión universitaria y sociología. Publicaciones recientes: "La responsabilidad social universitaria en la dirección estratégica de las universidades" (2017), "Nueva gobernanza universitaria: enfoque omniabarcante para estudiar el gobierno en universidades latinoamericanas" (2018), "Gobernanza universitaria y valores: la función control en la gestión universitaria” (2018), "Tipología de los sistemas de aseguramiento de la calidad basado en la autonomía y la Responsabilidad Social Universitaria” (2020).
\end{abstract}




\section{Referencias}

Almuiñas, J. y Galarza, J. (2015). Las alianzas estratégicas y sus singularidades en las relaciones de las Instituciones de Educación Superior y las empresas. Estrategia y gestión universitaria, 3(2), 56-67. http://revistas.unica.cu/index.php/regu/article/view/366

Alzate, F., Chaverra, L. y Arango, E. (2020). Gestión directiva universitaria desde el paradigma de la complejidad: otra mirada y nuevas propuestas. Revista Hallazgos, 17(33), 23-51. https://doi.org/10.15332/2422409X.5454

Alzate, J. y Cardona, J. (2018). La planeación gubernamental en la universidad pública. Elementos para el análisis crítico de los cambios en la gestión universitaria. En-Contexto, 6(8), 125-138. https://ojs.tdea.edu.co/index.php/encontexto/article/view/482

Ansoff, I. (1998). La dirección estratégica en la práctica empresarial. Addison Wesley Longman.

Ashby, E. (1963). Introduction: Decision-Making in the academic world. Sociological Studies in British University Education, Monograph No. 7. University of Keele.

Ballvé, A. (2000). Tablero de Control. Organizando información para crear valor. Macchi.

Berrios, O., Castillo, M. y Castro, E. (2009). Modelo gerencial en el marco de la transformación universitaria. Revista de Ciencias Sociales, 15(3), 519-528. http://www.scielo.org.ve/scielo. php?script=sci_arttext\&pid=S1315-95182009000300013\&lng=es\&tlng=es

Brunner, J. y Ganga, F. (2016). Dinámicas de transformación en la Educación Superior Latinoamericana: desafíos para la gobernanza. Revista Opción, 32(80), 12-35. https://www. redalyc.org/pdf/310/31047691002.pdf

Calderón, R. (2009). Ciencia, desarrollo humano y calidad de vida. Revista Hallazgos, 6(12), 4757. https://doi.org/10.15332/s1794-3841.2009.0012.02

De Melo, V. (2018). Análise interdiscursiva de políticas públicas: reflexão epistemológica. Revista Ámbito, 39, 1-14. https://revistascientificas.us.es/index.php/Ambitos/article/ view/9067

Dolan, S. (2012). Coaching por Valores. Una guía para los sucesos en la vida de los negocios o el negocio de la vida. Book 7.

Ganga, F. (2014). Gobernanza universitaria. Aproximaciones teóricasy empíricas. Editorial Cedac.

Ganga, F. y Núñez, O. (2018). Gobernanza de las organizaciones: acercamiento conceptual a las instituciones de Educación Superior. Revista Espacios, 39(17), 1-9. http://www.revistaespacios.com/a18v39n17/18391709.html

Ganga, F., Pérez, A. y Mansilla, J. (2018). Paradigmas emergentes en la gobernanza universitaria: una aproximación teórica. Utopía y Praxis Latinoamericana, 23(83), 123-136. http:// www.redalyc.org/articulo.oa?id=27957772012

Ginés, J. (2018). Universidad: mitos, modas y tendencias. Revista Iberoamericana de Educación Superior, 9(24), 3-16. https://doi.org/10.22201/iisue.20072872e.2018.24.262 
García, F. J. (2018). La universidad en la sociedad del conocimiento. [Proyectos Docentes, Universidad de Salamanca]. Repositorio Grial. http://repositorio.grial.eu/handle/grial/1191

Garzón, C. y Rodríguez, P. (2019). La gobernanza y su incidencia en los procesos de calidad en las instituciones de educación superior. Revista Boletín Redipe, 8(10), 111-124. https://doi. org/10.36260/rbr.v8i10.838

Hueso, M. (2019). Interpretando la sociedad del conocimiento desde una perspectiva histórica de las sociedades humanas. Antrópica. Revista de Ciencias Sociales y Humanidades, 5(9), 77-92. https://antropica.com.mx/ojs/index.php/AntropicaRCSH/article/view/31

Kaplan, R. y Norton, D. (2000). El Cuadro de Mando Integral. Gestión 2000.

Kaplan, R. y Norton, D. (2001). Cómo utilizar el Cuadro de Mando Integral para implantary gestionar su estrategia. Gestión 2000.

Kaplan, R. y Norton, D. (2002). Cuadro de Mando Integral. (2.a edición). Gestión 2000.

Kaplan, R. y Norton, D. (2004). Mapas estratégicos. Gestión 2000.

Kaplan, R. y Norton, D. (2006). Alignment. Incrementando los resultados mediante el alineamiento estratégico en toda la organización. Harvard Business School Press.

Knobel, M. y Bernasconi, A. (2017). Latin American Universities: Stuck in the Twentieth Century. International Higher Education, 88, 26-28. https://doi.org/10.6017/ihe.2017.88.9693

Krüger, K., Parellada, M., Samoilovich, D. y Sursock, A. (2019). Implementando las reformas de la gobernanza: las reglas de juego en los sistemas universitarios europeos. Revista de Educación, 385. 11-37. 10.4438/1988-592X-RE-2019-385-415

Laguna, S., Caballero, C., Lewis, V., Mazuera, S., Salamanca, J., Daza, W. y Fourzali, A. (2007). Consideraciones éticas en la publicación de investigaciones científicas. Salud Uninorte, 23(1), 64-78. http://rcientificas.uninorte.edu.co/index.php/salud/article/view/4055

Maldonado, B., Buenaño, J. y Benavides, K. (2019). Aproximación a un modelo de gobernanza en universidades públicas de la provincia de Pichincha del Ecuador. Visión de Futuro, Año $16,23(2), 102-121$.

Mendoza, J. (2011). El día de la universidad. Procesos Históricos. Revista de Historia y Ciencias Sociales, 20, 185-194.

Murcia, N. y Gamboa, A. (2014). Saber, manipulación y poder. La universidad como institución social. Revista Hallazgos, 11(22), 419-434. https://doi.org/10.15332/s17943841.2014.0022.21

Naranjo, R., Mesa, M. y Solera, J. (2005). El control estratégico. Lo que no debemos obviar. Revista Tecnología en Marcha, 18(4), 3-8. https://revistas.tec.ac.cr/index.php/tec_marcha/ article/view/7

Olve, N., Roy, J. y Wetter, M. (2002). Implantando y gestionando el Cuadro de Mando Integral: Guía práctica del Balanced Scorecard. Gestión 2000. 
Organización de las Naciones Unidas para la Educación, la Ciencia y la Cultura [Unesco] (2011). La Filosofía: Una escuela de la Libertad. Unesco. https://unesdoc.unesco.org/ark:/48223/ pfo000192689

Organización de las Naciones Unidas para la Educación, la Ciencia y la Cultura [Unesco] (1998). Declaración Mundial sobre la Educación Superior en el siglo xxi: visión y acción. Conferencia Mundial sobre Educación Superior. https://unesdoc.unesco.org/ark:/48223/ pf0000113878_spa

Organización de las Naciones Unidas [ONU]. (2015). Objetivos y metas de desarrollo sostenible, Agenda2030deDesarrollo Sostenible.onu.https://www.un.org/sustainabledevelopment/ es/objetivos-de-desarrollo-sostenible/

Pérez, A. (2013). El sistema de calidad basado en valores. [Tesis inédita de doctorado]. Universidad de Camagüey Ignacio Agramonte Loynaz.

Pérez, A., Aguilar, J. y Rodríguez, A. (2018). Nueva gobernanza universitaria: enfoque omniabarcante para estudiar el gobierno en universidades latinoamericanas. Revista Venezolana de Gerencia, 23(E.E.1), 53-66. https://www.redalyc.org/articulo.oa?id=29062781004

Pérez, A., Naranjo, R. y Mesa, M. (2017). La responsabilidad social universitaria en la dirección estratégica de las universidades. Revista Estrategia y Gestión Universitaria, 5(1),7790. http://revistas.unica.cu/index.php/regu/article/view/603/720

Peters, T. y Waterman, R. (1986). En busca de la excelencia. Lecciones de las empresas mejor gestionadas de EE.UU. Folio, S.A.

Ronda, G. (2007). Dirección estratégica, constructos y dimensiones. Futuro.

Samoilovich, D. (2007). Escenarios de gobierno en las universidades europeas. Fundación CyD.

Sánchez, A. A. (2011). Manual de redacción académica y científica: cómo escribir, evaluar y publicar artículos. Fundación Universitaria Católica del Norte.

Sánchez, M. y Castañón, J. (2019). Gobernanza en la educación superior de México. Caso Universidad Autónoma de Tamaulipas. Revista de Ciencias Sociales, 25(3), 59-71. https://produccioncientificaluz.org/index.php/rcs/article/view/27356/28032

Scott, R. (2014). Institutions and organizations. Ideas, interests, and identities. SAGE.

Stoner, J. y Freeman, R. (1994). Administración. (5. ${ }^{a}$ edición). Prentice Hall Hispanoamericana.

Viancos, P. y Ganga, F. (2020). Propuesta teórica de taxonomía de universidades iberoamericanas a partir de la forma de nombramiento de los rectores. Revista propósitos y representaciones, 8(SPE1), e501. http://dx.doi.org/10.20511/pyr2020.v8nSPE1.501

Yera, R., Septién, L. y Naranjo, R. (2019). Aplicación informática de soporte a la gestión estratégica. Revista Retos de la Dirección, 13(2), 258-283. http://scielo.sld.cu/pdf/rdir/ v13n2/2306-9155-rdir-13-02-258.pdf 